\title{
DSDP5 User Guide - Software for Semidefinite Programming
}

prepared by

Mathematics and Computer Science Division Argonne National Laboratory 


\section{About Argonne National Laboratory}

Argonne is managed by The University of Chicago for the U.S. Department of Energy under contract W-31-109-Eng-38. The Laboratory's main facility is outside Chicago, at 9700 South Cass Avenue, Argonne, Illinois 60439. For information about Argonne and its pioneering science and technology programs, see www.anl.gov.

\section{Availability of This Report}

This report is available, at no cost, at http://www.osti.gov/bridge. It is also available on paper to U.S. Department of Energy and its contractors, for a processing fee, from:

U.S. Department of Energy

Office of Scientific and Technical Information

P.O. Box 62

Oak Ridge, TN 37831-0062

phone (865) 576-8401

fax (865) 576-5728

reports@adonis.osti.gov

\section{Disclaimer}

This report was prepared as an account of work sponsored by an agency of the United States Government. Neither the United States Government nor any agency thereof, nor The University of Chicago, nor any of their employees or officers, makes any warranty, express or implied, or assumes any legal liability or responsibility for the accuracy, completeness, or usefulness of any information, apparatus, product, or process disclosed, or represents that its use would not infringe privately owned rights. Reference herein to any specific commercial product, process, or service by trade name, trademark, manufacturer, or otherwise, does not necessarily constitute or imply its endorsement, recommendation, or favoring by the United States Government or any agency thereof. The views and opinions of document authors expressed herein do not necessarily state or reflect those of the United States Government or any agency thereof, Argonne National Laboratory, or The University of Chicago. 


\section{DSDP5 User Guide - Software for Semidefinite Programming}

by S.J.Benson and Y.Ye

Mathematics and Computer Science Division

Technical Memorandum No. 277

Argonne National Laboratory

September 2005

This work was supported by the Mathematical, Information, and Computational Sciences

Division subprogram of the Office of Advanced Scientific Computing Research, Office of Science, U.S. Department of Energy, under Contract W-31-109-Eng-38.

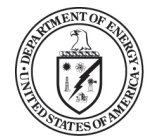




\section{Contents}

$\begin{array}{ll}\text { Abstract } & 1\end{array}$

1 Notation 2

2 Dual-Scaling Algorithm 3

3 Standard Form 5

4 Iteration Monitor $\quad 7$

5 DSDP with MATLAB $\quad 8$

5.1 Semidefinite Cones . . . . . . . . . . . . . . . . . 8

5.2 LP Cones . . . . . . . . . . . . . . . . . . . . . . 9

5.3 Solver Options . . . . . . . . . . . . . . . . . . . . . . . 10

5.4 Solver Performance and Statistics . . . . . . . . . . . . . . . . . 11

6 Reading SDPA Files $\quad 13$

7 Applying DSDP to Graph Problems 14

8 DSDP Subroutine Library $\quad 15$

8.1 Creating the Solver . . . . . . . . . . . . . . . . . 15

8.2 Semidefinite Cone . . . . . . . . . . . . . . . . . . 15

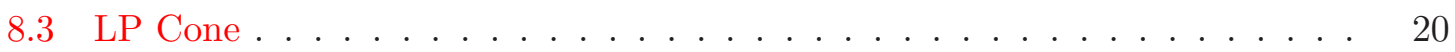

8.4 Applying the Solver . . . . . . . . . . . . . . . . . . 22

8.5 Convergence Criteria . . . . . . . . . . . . . . . . . . 23

8.6 Detecting Infeasibility . . . . . . . . . . . . . . . . . . 24

8.7 Solutions and Statistics _. . . . . . . . . . . . . . . . 24

8.8 Improving Performance . . . . . . . . . . . . . . . . . 26

8.9 Iteration Monitor . . . . . . . . . . . . . . . . 27

9 PDSDP $\quad 28$

10 Data Structures and Parameters $\quad 30$

11 Previous Versions $\quad 33$

$\begin{array}{ll}\text { Acknowledgments } & 33\end{array}$

$\begin{array}{ll}\text { References } & 34\end{array}$ 


\title{
DSDP5 User Guide - Software for Semidefinite Programming
}

\author{
by \\ Steven J. Benson and Yinyu Ye
}

\begin{abstract}
DSDP implements the dual-scaling algorithm for semidefinite programming. The source code of this interior-point solver, written entirely in ANSI C, is freely available. The solver can be used as a subroutine library, as a function within the Matlab environment, or as an executable that reads and writes to files. Initiated in 1997, DSDP has developed into an efficient and robust general-purpose solver for semidefinite programming. Although the solver is written with semidefinite programming in mind, it can also be used for linear programming and other constraint cones.
\end{abstract}

The features of DSDP include the following:

- a robust algorithm with a convergence proof and polynomially bounded complexity under mild assumptions on the data,

- primal and dual solutions,

- feasible solutions when they exist or approximate certificates of infeasibity,

- initial points that can be feasible or infeasible,

- relatively low memory requirements for an interior-point method,

- sparse and low-rank data structures,

- extensibility that allows applications to customize the solver and improve its performance,

- a subroutine library that enables it to be linked to larger applications,

- scalable performance for large problems on parallel architectures, and

- a well-documented interface and examples of its use.

The package has been used in many applications and tested for efficiency, robustness, and ease of use. We welcome and encourage further use under the terms of the license included in the distribution. 


\section{Notation}

The DSDP package implements a dual-scaling algorithm to find solutions $\left(X_{j}, y_{i}, S_{j}\right)$ to linear and semidefinite optimization problems of the form

$$
\begin{aligned}
& (P) \quad \inf \sum_{j=1}^{p}\left\langle C_{j}, X_{j}\right\rangle \quad \text { subject to } \sum_{j=1}^{p}\left\langle A_{i, j}, X_{j}\right\rangle=b_{i}, \quad i=1, \ldots, m, \quad X_{j} \in K_{j}, \\
& (D) \quad \sup \sum_{i=1}^{m} b_{i} y_{i} \quad \text { subject to } \sum_{i=1}^{m} A_{i, j} y_{i}+S_{j}=C_{j}, \quad j=1, \ldots, p, \quad S_{j} \in K_{j} .
\end{aligned}
$$

In this formulation, $b_{i}$ and $y_{i}$ are real scalars.

For semidefinite programming, the data $A_{i, j}$ and $C_{j}$ are symmetric matrices of dimension $n_{j}\left(\mathbb{S}^{n_{j}}\right)$, and the cone $K_{j}$ is the set of symmetric positive semidefinite matrices of the same dimension. The inner product $\langle C, X\rangle:=C \bullet X:=\sum_{k, l} C_{k, l} X_{k, l}$, and the symbol $\succ(\succeq)$ means the matrix is positive (semi)definite. In linear programming, $A_{i}$ and $C$ are vectors of real scalars, $K$ is the nonnegative orthant, and the inner product $\langle C, X\rangle$ is the usual vector inner product.

More generally, users specify $C_{j}, A_{i, j}$ from an inner-product space $V_{j}$ that intersects a cone $K_{j}$. Using the notation summarized in Table 1, let the symbol $\mathcal{A}$ denote the linear map $\mathcal{A}: V \rightarrow \mathbb{R}^{m}$ defined by $(\mathcal{A} X)_{i}=\left\langle A_{i}, X\right\rangle$; its adjoint $\mathcal{A}^{T}: \mathbb{R}^{m} \rightarrow V$ is defined by $\mathcal{A}^{T} y=\sum_{i=1}^{m} y_{i} A_{i}$. Equivalent expressions for $(\mathrm{P})$ and $(\mathrm{D})$ can be written

$$
\begin{aligned}
& (P) \quad \inf \langle C, X\rangle \quad \text { subject to } \mathcal{A} X=b, \quad X \in K \text {, } \\
& \text { (D) } \sup b^{T} y \quad \text { subject to } \mathcal{A}^{T} y+S=C, \quad S \in K \text {. }
\end{aligned}
$$

Formulation (P) will be referred to as the primal problem, and formulation (D) will be referred to as the dual problem. Variables that satisfy the linear equations are called feasible, whereas the others are called infeasible. The interior of the cone will be denoted by $\hat{K}$, and the interior feasible sets of $(\mathrm{P})$ and $(\mathrm{D})$ will be denoted by $\mathcal{F}^{0}(P)$ and $\mathcal{F}^{0}(D)$, respectively.

Table 1: Terms and notation for linear (LP), semidefinite (SDP), and conic programming.

\begin{tabular}{lcccc} 
Term & LP & SDP & Conic & Notation \\
\hline Dimension & $n$ & $n$ & $\sum n_{j}$ & $n$ \\
Data Space $\left(\ni C, A_{i}\right)$ & $\mathbb{R}^{n}$ & $\mathbb{S}^{n}$ & $V_{1} \oplus \ldots \oplus V_{p}$ & $V$ \\
Cone & $x, s \geq 0$ & $X, S \succeq 0$ & $X, S \in K_{1} \oplus \ldots \oplus K_{p}$ & $X, S \in K$ \\
Interior of Cone & $x, s>0$ & $X, S \succ 0$ & $X, S \in \hat{K}_{1} \oplus \ldots \oplus \hat{K}_{p}$ & $X, S \in \hat{K}$ \\
Inner Product & $c^{T} x$ & $C \bullet X$ & $\sum\left\langle C_{j}, X_{j}\right\rangle$ & $\langle C, X\rangle$ \\
Norm & $\|x\|_{2}$ & $\|X\|_{F}$ & $\left(\sum\left\|X_{j}\right\|^{2}\right)^{1 / 2}$ & $\|X\|$ \\
Product & {$\left[x_{1} s_{1} \ldots x_{n} s_{n}\right]^{T}$} & $X S$ & $X_{1} S_{1} \oplus \ldots \oplus X_{p} S_{p}$ & $X S$ \\
Identity Element & {$[1 \ldots 1]^{T}$} & $I$ & $I_{1} \oplus \ldots \oplus I_{p}$ & $I$ \\
Inverse & {$\left[1 / s_{1} \ldots 1 / s_{n}\right]^{T}$} & $S^{-1}$ & $S_{1}^{-1} \oplus \ldots \oplus S_{p}^{-1}$ & $S^{-1}$ \\
Dual Barrier & $\sum \ln s_{j}$ & $\ln \operatorname{det} S$ & $\sum \ln \operatorname{det} S_{j}$ & $\ln \operatorname{det} S$
\end{tabular}




\section{Dual-Scaling Algorithm}

This section summarizes the dual-scaling algorithm for solving (P) and (D). For simplicity, parts of this discussion assume that the cone is a single semidefinite block, but an extension of the algorithm to multiple blocks and other cones is relatively simple. This discussion also assumes that the $A_{i}$ s are linearly independent, there exists $X \in \mathcal{F}^{0}(P)$, and a starting point $(y, S) \in \mathcal{F}^{0}(D)$ is known. The next section discusses how DSDP generalizes the algorithm to relax these assumptions.

It is well known that under these assumptions, both (P) and (D) have optimal solutions $X^{*}$ and $\left(y^{*}, S^{*}\right)$, which are characterized by the equivalent conditions that the duality gap $\left\langle X^{*}, S^{*}\right\rangle$ is zero and the product $X^{*} S^{*}$ is zero. Moreover, for every $\nu>0$, there exists a unique primal-dual feasible solution $\left(X_{\nu}, y_{\nu}, S_{\nu}\right)$ that satisfies the perturbed optimality equation $X_{\nu} S_{\nu}=\nu I$. The set of all solutions $\mathcal{C} \equiv\left\{\left(X_{\nu}, y_{\nu}, S_{\nu}\right): \nu>0\right\}$ is known as the central path, and $\mathcal{C}$ serves as the basis for path-following algorithms that solve (P) and (D). These algorithms construct a sequence $\{(X, y, S)\} \subset \mathcal{F}^{0}(P) \times \mathcal{F}^{0}(D)$ in a neighborhood of the central path such that the duality gap $\langle X, S\rangle$ goes to zero. A scaled measure of the duality gap that proves useful in the presentation and analysis of path-following algorithms is $\mu(X, S)=\langle X, S\rangle / n$ for all $(X, S) \in K \times K$. Note that for all $(X, S) \in \hat{K} \times \hat{K}$, we have $\mu(X, S)>0$ unless $X S=0$. Moreover, $\mu\left(X_{\nu}, S_{\nu}\right)=\nu$ for all points $\left(X_{\nu}, y_{\nu}, S_{\nu}\right)$ on the central path.

The dual-scaling algorithm applies Newton's method to $\mathcal{A} X=b, \mathcal{A}^{T} y+S=C$, and $X=\nu S^{-1}$ to generate

$$
\begin{aligned}
\mathcal{A}(X+\Delta X) & =b, \\
\mathcal{A}^{T}(\Delta y)+\Delta S & =0, \\
\nu S^{-1} \Delta S S^{-1}+\Delta X & =\nu S^{-1}-X .
\end{aligned}
$$

Equations (1) - (3) will be referred to as the Newton equations; their Schur complement is

$$
\nu\left(\begin{array}{ccc}
\left\langle A_{1}, S^{-1} A_{1} S^{-1}\right\rangle & \cdots & \left\langle A_{1}, S^{-1} A_{m} S^{-1}\right\rangle \\
\vdots & \ddots & \vdots \\
\left\langle A_{m}, S^{-1} A_{1} S^{-1}\right\rangle & \cdots & \left\langle A_{m}, S^{-1} A_{m} S^{-1}\right\rangle
\end{array}\right) \Delta y=b-\nu \mathcal{A} S^{-1} .
$$

The left-hand side of this linear system is positive definite when $S \in \hat{K}$. In this manuscript, it will sometimes be referred to as $M$. DSDP computes $\Delta^{\prime} y:=M^{-1} b$ and $\Delta^{\prime \prime} y:=M^{-1} \mathcal{A} S^{-1}$. For any $\nu$,

$$
\Delta_{\nu} y:=\frac{1}{\nu} \Delta^{\prime} y-\Delta^{\prime \prime} y
$$

solves (4). We use the subscript to emphasize that $\nu$ can be chosen after computing $\Delta^{\prime} y$ and $\Delta^{\prime \prime} y$ and that the value chosen for the primal step may be different from the value chosen for the dual step.

Using (2),(3), and $\Delta_{\nu} y$, we get

$$
X(\nu):=\nu\left(S^{-1}+S^{-1}\left(\mathcal{A}^{T} \Delta_{\nu} y\right) S^{-1}\right),
$$

which satisfies $\mathcal{A} X(\nu)=b$. Because $X(\nu) \in \hat{K}$ if and only if

$$
C-\mathcal{A}^{T}\left(y-\Delta_{\nu} y\right) \in \hat{K},
$$


DSDP applies a Cholesky factorization on (6) to test the condition. If $X(\nu) \in \hat{K}$, a new upper bound

$$
\bar{z}:=\langle C, X(\nu)\rangle=b^{T} y+\langle X(\nu), S\rangle=b^{T} y+\nu\left(\Delta_{\nu} y^{T} \mathcal{A} S^{-1}+n\right)
$$

can be obtained without explicitly computing $X(\nu)$. The dual-scaling algorithm does not require $X(\nu)$ to compute the step direction defined by (4), so DSDP does not compute it unless specifically requested. This feature characterizes the algorithm and its performance.

To get closer to the central path and make further use of $M$, whose computation and factorization usually dominate the computation time, DSDP generates a sequence of corrector steps. The corrector steps compute $\mathcal{A} S^{-1}$ using the current $S$ and $\Delta^{c} y:=M^{-1} \mathcal{A} S^{-1}$. Since the computation of $M$ used previous values of $S$, the corrector step

$$
\Delta_{\nu}^{c} y:=\frac{1}{\nu} \Delta^{\prime} y-\Delta^{c} y
$$

is not a Newton step. A line search computes a step length, $\alpha_{c}$, that improves the merit function

$$
\phi_{\nu}(y):=b^{T} y+\nu \ln \operatorname{det} S .
$$

Between 0 and 12 corrector steps are applied each iteration. The exact number can be chosen heuristically based on the square of the ratio of $m$ and the dimension of the largest semidefinite block.

Either $(y, S)$ or $X$ reduces the dual potential function

$$
\psi(y):=\rho \log \left(\bar{z}-b^{T} y\right)-\ln \operatorname{det} S
$$

enough at each iteration to achieve linear convergence. 


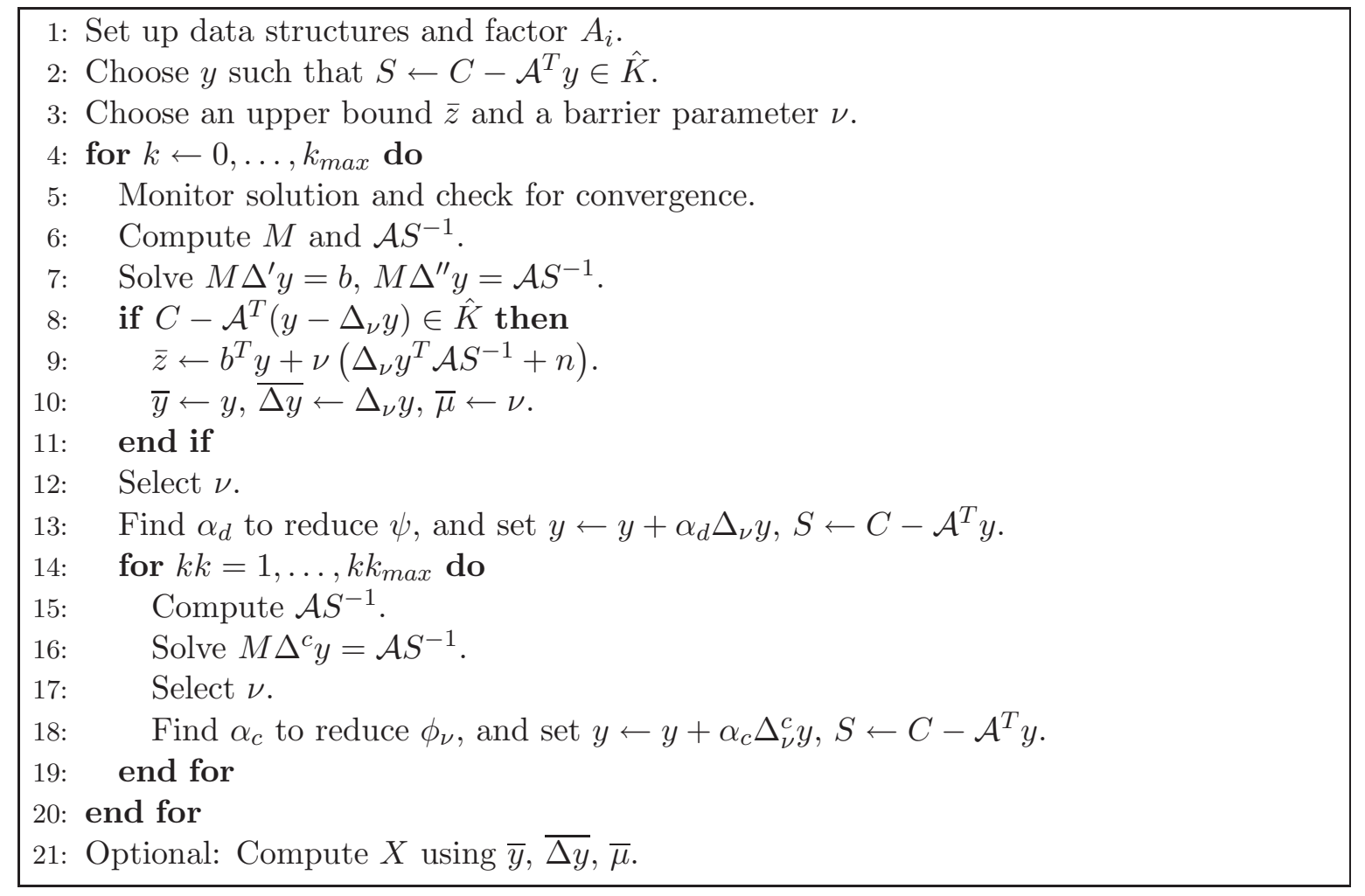

\section{Standard Form}

The convergence of the algorithm assumes that both (P) and (D) have an interior feasible region and the current solutions are elements of the interior. To satisfy these assumptions, DSDP bounds the variables $y$ such that $l \leq y \leq u$, where $l, u \in \mathbb{R}^{m}$. By default, $l_{i}=-10^{7}$ and $u_{i}=10^{7}$ for each $i$ from 1 through $m$. Furthermore, DSDP bounds the trace of $X$ by a penalty parameter $\Gamma$ whose default value is $\Gamma=10^{8}$. Including these bounds and their associated Lagrange variables $x^{l} \in \mathbb{R}^{m}, x^{u} \in \mathbb{R}^{m}$, and $r$, DSDP solves the following pair of problems:

$$
\begin{aligned}
& (P P) \quad \text { minimize }\langle C, X\rangle+u^{T} x^{u}-l^{T} x^{l} \\
& \text { subject to } \mathcal{A} X+x^{u}-x^{l}=b \text {, } \\
& \langle I, X\rangle \leq \Gamma, \\
& X \in K, \quad x^{u} \geq 0, \quad x^{l} \geq 0 . \\
& (D D) \quad \text { maximize } \quad b^{T} y-\Gamma r \\
& \text { subject to } C-\mathcal{A}^{T} y+I r=S \in K \text {, } \\
& l \leq y \leq u, \quad r \geq 0 .
\end{aligned}
$$

The reformulations (PP) and (DD) are bounded and feasible, so the optimal objective values to this pair of problems are equal. Furthermore, (PP) and (DD) can be expressed in the form of $(\mathrm{P})$ and $(\mathrm{D})$.

Unless the user provides a feasible point $y$, DSDP uses the $y$ values provided by the application (usually all zeros) and increases $r$ until $C-\mathcal{A}^{T} y+\operatorname{Ir} \in \hat{K}$. Large values of $r$ 
improve robustness, but smaller values often improve performance. In addition to bounding $X$, the parameter $\Gamma$ penalizes infeasiblity in (D) and forces $r$ toward zero. The nonnegative variable $r$ increases the dimension $m$ by one and adds an inequality to the original problem. The $M$ matrix treats $r$ separately by storing the corresponding row/column as a separate vector and applying the Sherman-Morrison-Woodbury formula. Unlike other inequalities, DSDP allows $r$ to reach the boundary of the cone. Once $r=0$, it is fixed and effectively removed from the problem.

The bounds on $y$ add $2 m$ inequality constraints to the original problem; and, with a single exception, DSDP treats them the same as the constraints on the original model. One difference between these bounds and the other constraints is that DSDP explicitly computes the corresponding Lagrangian variables $x^{l}$ and $x^{u}$ at each iteration to quantify the infeasibility in $(\mathrm{P})$. The bounds $l$ and $u$ penalize infeasibility in $(\mathrm{P})$, force $x^{l}$ and $x^{u}$ toward zero, and prevent numerical difficulties created by variables with large magnitude.

The solution to (PP) and (DD) is a solution to (P) and (D) when the optimal objective values of $(\mathrm{P})$ and $(\mathrm{D})$ exist and are equal and the bounds are sufficiently large. DSDP identifies unboundedness or infeasibility in $(\mathrm{P})$ and $(\mathrm{D})$ through examination of the solutions to $(\mathrm{PP})$ and $(\mathrm{DD})$. Given parameters $\epsilon_{P}$ and $\epsilon_{D}$,

- if $r \leq \epsilon_{r},\|\mathcal{A} X-b\|_{\infty} /\langle I, X\rangle>\epsilon_{P}$, and $b^{T} y>0$, it characterizes (D) as unbounded and $(\mathrm{P})$ as infeasible;

- if $r>\epsilon_{r}$ and $\|\mathcal{A} X-b\|_{\infty} /\langle I, X\rangle \leq \epsilon_{P}$, it characterizes (D) as infeasible and (P) as unbounded.

Normalizing unbounded solutions will provide an approximate certificate of infeasibility. Larger bounds may improve the quality of the certificate of infeasibility and permit additional feasible solutions, but they may also create numerical difficulties in the solver. 


\section{Iteration Monitor}

The progress of the DSDP solver can be monitored by using standard output printed to the screen. The data below shows an example of this output.

\begin{tabular}{|c|c|c|c|c|c|c|c|c|}
\hline Iter & PP Objective & DD Objective & PInfeas & DInfeas & $\mathrm{Nu}$ & \multicolumn{2}{|c|}{ StepLength } & Pnrm \\
\hline 0 & $1.00000000 \mathrm{e}+02$ & $-1.13743137 e+05$ & $2.2 e+00$ & $3.8 e+02$ & $1.1 \mathrm{e}+05$ & 0.00 & 0.00 & 0.00 \\
\hline & $1.36503342 \mathrm{e}+06$ & $-6.65779055 e+04$ & $5.1 e+00$ & $2.2 e+02$ & $1.1 e+04$ & 1.00 & 0.33 & 4.06 \\
\hline & $1.36631922 \mathrm{e}+05$ & $-6.21604409 e+$ & $5.4 e^{+}$ & $1.9 e+01$ & $4.5 e+02$ & 1.00 & .00 & 7.85 \\
\hline & 5.457991 & -3.182920 & $1.5 e$ & $9.1 e+00$ & $7.5 e+01$ & 1.00 & 1.00 & 17.63 \\
\hline & 1.02930 & -5.391661 & $1.1 \mathrm{e}$ & $5.3 e-01$ & $2.7 e+01$ & 1.00 & 1.00 & 7.58 \\
\hline & $4.30074471 e+02$ & $-3.02460061 e+01$ & $3.3 e-09$ & $0.0 e+00$ & $5.6 e+00$ & 1.00 & 1.00 & 11.36 \\
\hline & $8.99999824 \mathrm{e}+00$ & $8.99999617 e+00$ & $1.1 e-16$ & & $1.7 e-08$ & & 1.0 & 7.03 \\
\hline & $8.99999668 e+00$ & $8.99999629 e+00$ & $2.9 e-19$ & $0.0 e+00$ & $3.4 e-09$ & 1.00 & 1.00 & 14.19 \\
\hline
\end{tabular}

The program will print a variety of statistics for each problem to the screen.

Iter the iteration number.

PP Objective the upper bound $\bar{z}$ and objective value in (PP).

DD Objective the objective value in (DD).

PInfeas the primal infeasiblity in $(\mathrm{P})$ is $\left\|x^{u}-x^{l}\right\|_{\infty}$.

DInfeas the dual infeasibility in (D) is the variable $r$.

$\mathrm{Nu}$ the barrier parameter $\nu$.

StepLength the multiple of the step-directions in (P) and (D).

Pnrm the proximity to the central path: $\|\nabla \psi\|_{M^{-1}}$. 


\section{DSDP with MATLAB}

Additional help using the DSDP can be found by typing help dsdp in the directory DSDP5.X. The command

$>[\operatorname{STAT}, \mathrm{y}, \mathrm{X}]=\operatorname{dsdp}(\mathrm{b}, \mathrm{AC})$

attempts to solve the semidefinite program by using a dual-scaling algorithm. The first argument is the objective vector $b$ in (D) and the second argument is a cell array that contains the structure and data for the constraint cones. Most data has a block structure, which should be specified by the user in the second argument. For a problem with $p$ cones of constraints, AC is a $p \times 3$ cell array. Each row of the cell array describes a cone. The first element in each row of the cell array is a string that identifies the type of cone. The second element of the cell array specifies the dimension of the cone, and the third element contains the cone data.

\subsection{Semidefinite Cones}

If the cone $j$ is a semidefinite cone consisting of a single block with $n$ rows and columns in the matrices, then the first element in this row of the cell array is the string 'SDP' and the second element is the number $\mathrm{n}$, and the third element is a sparse matrix with $n(n+1) / 2$ rows and $m+1$ columns. Columns 1 to $m$ of this matrix represent the constraints $A_{1, j}, \ldots, A_{m, j}$ for this block, and column $m+1$ represents $C_{j}$.

The square symmetric data matrices $A_{i, j}$ and $C_{j}$ map to the columns of $\operatorname{AC}\{j, 3\}$ through the operator $\operatorname{dvec}(\cdot): \mathbb{R}^{n \times n} \rightarrow \mathbb{R}^{n(n+1) / 2}$, which is defined as

$$
\operatorname{dvec}(A)=\left[\begin{array}{llllllll}
a_{1,1} & a_{1,2} & a_{2,2} & a_{1,3} & a_{2,3} & a_{3,3} & \ldots & a_{n, n}
\end{array}\right]^{T} .
$$

In this definition, $a_{k, l}$ is the element in row $k$ and column $l$ of $A$. This ordering is often referred to as symmetric packed storage format. The inverse of $\operatorname{dvec}()$ is $\operatorname{dmat}(\cdot)$ : $\mathbb{R}^{n(n+1) / 2} \rightarrow \mathbb{R}^{n \times n}$, which converts the vector into a square symmetric matrix. Using these operations, we obtain

$$
A_{i, j}=\operatorname{dmat}(\operatorname{AC}\{j, 3\}(:, \mathrm{i})), \quad C_{j}=\operatorname{dmat}(\operatorname{AC}\{\mathrm{j}, 3\}(:, \mathrm{m}+1))
$$

and

$$
\operatorname{AC}\{j, 3\}=\left[\begin{array}{llll}
\operatorname{dvec}\left(A_{1, j}\right) & \ldots & \operatorname{dvec}\left(A_{m, j}\right) & \operatorname{dvec}\left(C_{j}\right)
\end{array}\right] ;
$$

For example, the problem

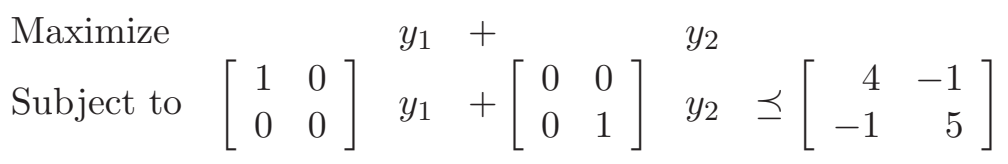

can be solved by

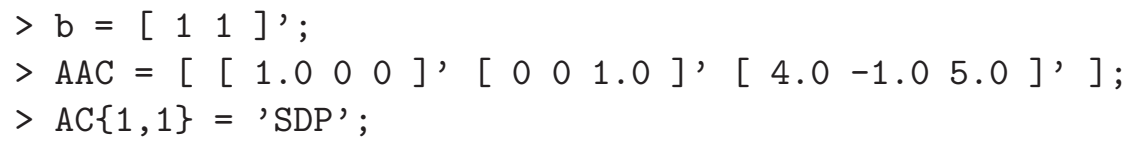




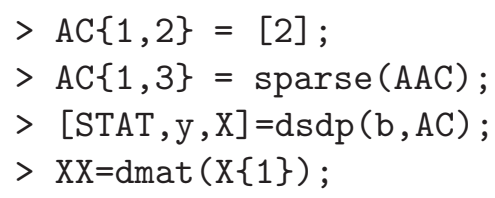

The solution $y$ is the column vector $\mathrm{y}^{\prime}=\left[\begin{array}{ll}3 & 4\end{array}\right]^{\prime}$, and the solution $X$ is a $p \times 1$ cell array. In this example, the solution $X=\left[\begin{array}{llll}3 \times & 1 & \text { double }\end{array}\right]$ is $X\{1\}^{\prime}=\left[\begin{array}{llll}1.0 & 1.0 & 1.0\end{array}\right]$. Furthermore, $\operatorname{dmat}(\mathrm{X}\{1\})=\left[\begin{array}{ccccc}1 & 1 & ; & 1 & 1\end{array}\right]$.

Each semidefinite block can be stated in a separate row of the cell array; only the available memory on the machine limits the number of cones that can be specified.

Each semidefinite block may, however, be grouped into a single row in the cell array. In order to group these blocks together, the second cell entry must be an array of integers stating the dimension of each block. The data from the blocks should be concatenated such that the number of rows in the data matrix increases whereas the number of columns remains constant. The following lines indicate how to group the semidefinite blocks in rows 1 and 2 of cell array AC1 into a new cell array AC2:

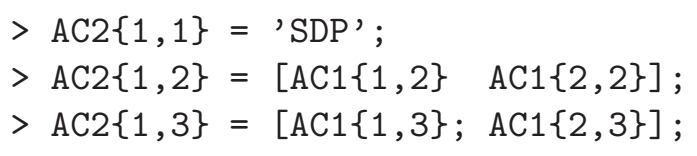

The new cell array AC2 can be passed directly into DSDP. The advantage of grouping multiple blocks together is that it uses less memory - especially when there are many blocks and many of the matrices in these blocks are zero. The performance of DSDP, measured by execution time, will change very little.

This distribution contains several examples files in SDPA format. A utility routine called readsdpa ( • ) can read these files and put the problems in DSDP format. They may serve as examples of how to format an application for use by the DSDP solver. Another example can be seen in the file maxcut ( . ), which takes a graph and creates an SDP relaxation of the maximum cut problem from a graph.

\subsection{LP Cones}

A cone of LP variables can specified separately. For example a randomly generated LP cone $A^{T} y \leq c$ with 3 variables $y$ and 5 inequality constraints can be specified in the following code.

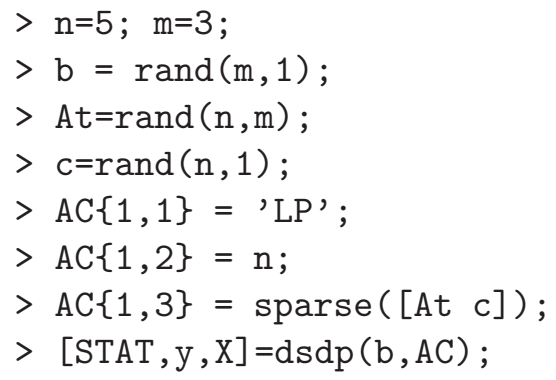

Multiple cones of LP variables may be passed into the DSDP solver, but for efficiency reasons, it is best to group them all together. This cone may also be passed to the DSDP solver as 
a semidefinite cone, where the matrices $A_{i}$ and $C$ are diagonal. For efficiency reasons, however, it is best to identify them separately as belonging to the cone of 'LP' variables.

Although $y$ variables that are fixed to a constant can be preprocessed and removed from a model, it is often more convenient to leave them in the model. It is more efficient to identify fixed variables to DSDP than to model these constraints as a pair of linear inequalities. The following example sets variables 1 and 8 to the values 2.4 and -6.1 , respectively.

$>\operatorname{AC}\{j, 1\}={ }^{\prime} F I X E D^{\prime} ; \operatorname{AC}\{j, 2\}=\left[\begin{array}{ll}1 & 8\end{array}\right] ; \operatorname{AC}\{j, 3\}=\left[\begin{array}{ll}2.4 & -6.1\end{array}\right] ;$

The corresponding variables $x$ to these constraints may be positive or negative.

\subsection{Solver Options}

There are more ways to call the solver. The command

$>[\mathrm{STAT}, \mathrm{y}, \mathrm{X}]=\operatorname{DSDP}(\mathrm{b}, \mathrm{AC}, \mathrm{OPTIONS})$

specifies some options for the solver. The OPTIONS structure may contain any of the following fields that may significantly affect the performance of the solver. The following options affect the formulation of the problem:

ro initial value for $r$ in (DD). If $r 0<0$, a heuristic will select a very large number $(\sim 1 \mathrm{e} 10)$. To improve convergence, try a smaller value. [default -1 (Heuristic)].

zbar an upper bound $\bar{z}$ on the objective value at the solution [default $1.0 \mathrm{e} 10]$.

penalty penalty parameter $\Gamma$ in (DD) that enforces feasibility in (D). IMPORTANT: This parameter must be positive and greater than the trace of the solution $X$ of $(\mathrm{P})$. [default 1e8].

boundy determines the bounds $l$ and $u$ on the variables $y$ in (DD). That is, -boundy $=l \leq y_{i} \leq u=$ boundy for all $i$. [default: $1 \mathrm{e} 7$.

The following fields in the OPTIONS structure affect the stopping criteria for the solver:

gaptol tolerance for duality gap as a fraction of the value of the objective functions [default 1e-6].

maxit maximum number of iterations allowed [default 1000].

steptol tolerance for stopping because of small steps [default 1e-2].

pnormtol $\|P(\nu)\|$ of solution should also be less than [default 1e30].

inftol the value $r$ in (DD) must be less than this tolerance to classify the final solution of (D) as feasible. [default 1e-8].

dual_bound Terminate the solver when it finds a feasible point of (D) with an objective greater than this value. (Helpful in branch-andbound algorithms.) [default $1 \mathrm{e}+30]$.

The following fields in the OPTIONS structure affect printing: 
print $=\mathrm{k}$ to display output at each $\mathrm{k}$ iteration, else $=0$ [default $10]$.

logtime $=1$ to profile the performance of DSDP subroutines, else $=0$. (Assumes proper compilation flags.)

cc add this constant the objective value. This parameter is algorithmically irrelevant, but it can make the objective values displayed on the screen more consistent with the underlying application [default 0].

Other fields are also recognized in OPTIONS structure:

rho to set the potential parameter $\rho$ in the function (9) to this multiple of the conic dimension $n$. [default: 3] IMPORTANT: Increasing this parameter to 4 or 5 may significantly improve performance.

dynamicrho to use dynamic rho strategy. [default: 1].

bigM if $>0$, the variable $r$ in (DD) will remain positive (as opposed to nonegative). [default 0 ].

mu0 initial barrier parameter $\nu$. [default -1: use heuristic]

reuse sets a maximum on the number of times the Schur complement matrix can be reused. Larger numbers reduce the number of iterations but increase the cost of each iteration. Applications requiring few iterations $(<60)$ should consider setting this parameter to 0 . [default: 4 ]

For instance, the commands

$>$ OPTIONS.gaptol $=0.001$

$>$ OPTIONS. boundy $=1000$;

$>$ OPTIONS.rho $=5$;

$>[\mathrm{STAT}, \mathrm{y}, \mathrm{X}]=\operatorname{DSDP}(\mathrm{b}, \mathrm{AC}$, OPTIONS $)$;

ask for a solution with approximately three significant digits, bound the $y$ variables by -1000 and +1000 , and use a potential parameter $\rho$ of 5 times the conic dimension. Some of these fields, especially rho, ro, and ybound, can significantly improve performance of the solver.

Using a fourth input argument, the command

$>[\operatorname{STAT}, \mathrm{y}, \mathrm{X}]=\operatorname{DSDP}(\mathrm{b}, \mathrm{AC}$, OPTIONS, $\mathrm{y} 0)$;

specifies an initial solution y0 in (D). The default starting vector is the zero vector.

\subsection{Solver Performance and Statistics}

The second and third output arguments return objective values for (D) and (P), respectively.

The first output argument is a structure with several fields that describe the solution of the problem: 
stype PDFeasible if the solutions to both (D) and (P) are feasible, Infeasible if (D) in infeasible, and and Unbounded if (D) is unbounded.

obj an approximately optimal objective value.

pobj objective value of (PP).

dobj objective value of (DD).

stopcode equals 0 if the solutions to (PP) and (DD) satisfy the prescribed tolerances and equals nonzero if the solver terminated for other reasons.

Additional fields describe characteristics of the solution:

tracex the trace of the solution $X$ of $(\mathrm{P})$.

$r$ the multiple of the identity element added to $C-\mathcal{A}^{T}(y)$ in the final solution to make $S$ positive definite.

$\mathrm{mu}$ the final barrier parameter $(\nu)$.

ynorm the largest element of y (infinity norm).

boundy the bounds placed on the magnitude of each variable $y$.

penalty the penalty parameter $\Gamma$ used by the solver, which must be greater than the trace of the variables $X$ in $(\mathrm{P})$. (see above).

Additional fields provide statistics from the solver:

iterations number of iterations used by the algorithm.

pstep the final step length in (PP)

dstep the final step length in (DD).

pnorm the final value $\|P(\nu)\|$.

rho the potential parameter (as a multiple of the total dimension of the cones).

gaphist a history of the duality gap.

infhist a history of the variable $r$ in (DD).

datanorm the Frobenius norm of C, A and b.

DSDP has also provides several utility routines. The utility derror ( · ) verifies that the solution satisfies the constraints and that the objective values $(\mathrm{P})$ and $(\mathrm{D})$ are equal. The errors are computed according to the the standards of the DIMACS Challenge. 


\section{Reading SDPA Files}

DSDP can be used if the user has a problem written in sparse SDPA format. These executables have been put in the directory DSDPROOT/exec/. The file name should follow the executable. For example,

$>$ dsdp5 truss4.dat-s

Other options can also be used with DSDP. These should follow the SDPA filename.

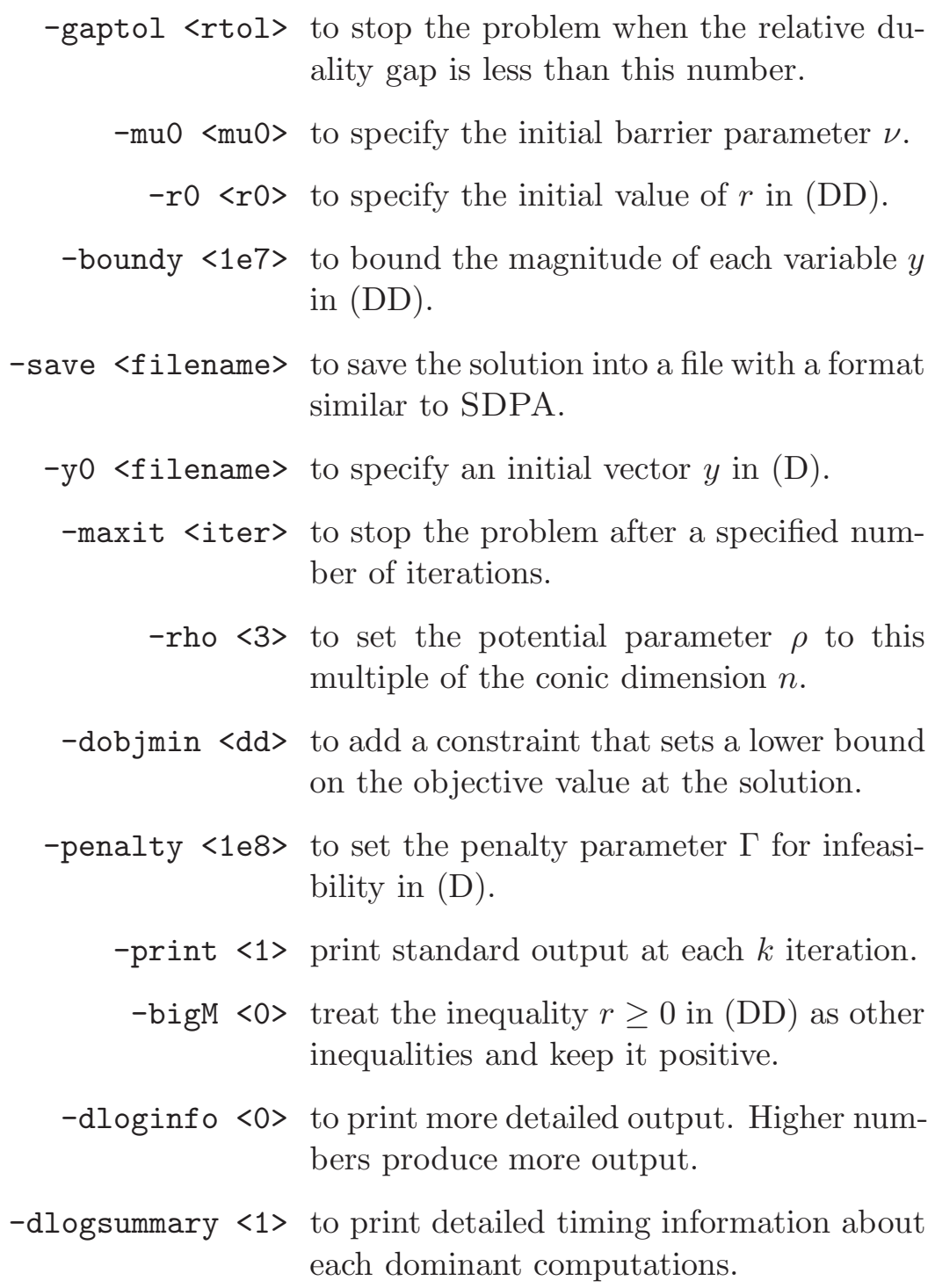




\section{Applying DSDP to Graph Problems}

Within the directory DSDPROOT/examples/ is a program maxcut.c that reads a file containing a graph, generates the semidefinite relaxation of a maximum cut problem, and solves the relaxation. For example,

$>$ maxcut graph1

reads the graph in the file graph1 and solves this graph problem. The first line of the graph should contain two integers. The first integer states the number of nodes in the graph, and the second integer states the number of edges. Subsequent lines have two or three entries separated by a space. The first two entries specify the two nodes that an edge connects. The optional third entry specifies the weight of the node. If no weight is specified, a weight of 1 will be assigned.

The same options that apply to reading SDPA files also apply here. Similar examples for the Lovász $\theta$ problem, maximum stable set problems, minimum graph coloring problem read also read a graph from a file, formulate the semidefinite relaxation, and solve it. 


\section{DSDP Subroutine Library}

DSDP can also be used within a $\mathrm{C}$ application through a set of subroutines. There are several examples of applications that use the DSDP application program interface. Within the DSDPROOT/examples/ directory, the file dsdp.c is a mex function that reads data from the Matlab environment, passes the data to the solver, and returns the solution. The file readsdpa.c reads data from a file for data in SDPA format, passes the data to the solver, and prints the solution. The files maxcut. $c$ and theta. $c$ read a graph, formulate a semidefinite relaxation to a combinatorial problem, and pass the data to a solver. The subroutines used in these examples are described in this chapter. Futher documentation on the routines and examples can be found in the HTML manual pages in DSDPROOT/docs/dox/html/.

Each of these applications includes the header file DSDPROOT/include/dsdp5.h and links to the library DSDPROOT/lib/libdsdp.a. All DSDP subroutines also return an int that represents an error code. A return value of zero indicates success, whereas a nonzero return value indicates that an error has occurred. The documentation of DSDP subroutines in this chapter will not show the return integer, but we highly recommend that applications check for errors after each subroutine.

\subsection{Creating the Solver}

To use DSDP through subroutines, first create a solver object with

int DSDPCreate(int $\mathrm{m}, \mathrm{DSDP}$ *newsolver);

The first argument in this subroutine is the number of variables in the problem. The second argument should be the address of a DSDP variable. The DSDP class is defined as a pointer to an internal structure that contains the state of the solver. This subroutine will construct a new structure and point the DSDP variable to a new solver. A difference in typeset distinguishes the DSDP software from the DSDP class. Objects of this class apply the dual-scaling algorithm to the data.

Specify the objective function associated with these variables using the subroutine

int DSDPSetDObjective(DSDP dsdp, int $i$, double bi);

The first argument is the solver, and the second and third arguments specify a variable number and the objective value $b_{i}$ associated with it. The variables are numbered 1 through $m$, where $m$ is the number of variables specified in DSDPCreate. The objective associated with each variable must be specified individually. The default value is zero.

The next step is to provide the conic structure and data in the problem. These subroutines will be described in the next sections.

\subsection{Semidefinite Cone}

To specify an application with a cone of semidefinite constraints, one can use the subroutine int DSDPCreateSDPCone(DSDP dsdp, int nblocks, SDPCone *newsdpcone); 
to create a new object that describes a semidefinite cone with one or more blocks. The first argument is an existing semidefinite solver, the second argument is the number of blocks in this cone, and the final argument is an address of a SDPCone variable. This subroutine will allocate structures needed to specify the constraints and point the variable to this structure. Multiple cones can be created for the same solver, but it is usually more efficient to group all semidefinite blocks into the same conic structure.

All subroutines that pass data to the semidefinite cone require an SDPCone object in the first argument. The second argument often refers to a specific block. The blocks will be labeled from 0 to nblocks-1. The subroutine int SDPConeSetBlockSize(SDPCone sdpcone, int blockj, int $\mathrm{n}$ ) can be used to specify the dimension of each block and the subroutine int SDPConeSetSparsity (SDPCone sdpcone, int blockj, int nnzmat) can be used to specify the number of nonzero matrices $A_{i, j}$ in each block. These subroutines are optional, but using them can improve error checking on the data matrices and perform a more efficient allocation of memory.

The data matrices can be specified by any of the following commands. The choice of data structures belongs to the user, and the performance of the problem depends upon this choice. In each of these subroutines, the first four arguments are an SDPCone object, the block number, the number of variable associated with it, and the number of rows and columns in the matrix. The blocks must be numbered consecutively, beginning with the number 0 . The $y$ variables are numbered consecutively from 1 to $m$. The objective matrices in $(\mathrm{P})$ are specified as constraint number 0. The data passed to the SDPCone object will be used in the solver but not modified. The user is responsible for freeing the arrays of data it passes to SDPCone after solving the problem.

The square symmetric data matrices $A_{i, j}$ and $C_{j}$ can be represented with a single array of numbers. DSDP supports the symmetric packed storage format. In symmetric packaged storage format, the elements of a matrix with $n$ rows and columns are ordered as follows:

$$
\left[\begin{array}{llllllll}
a_{1,1} & a_{2,1} & a_{2,2} & a_{3,1} & a_{3,2} & a_{3,3} & \ldots & a_{n, n}
\end{array}\right] .
$$

In this array $a_{k, l}$ is the element in row $k$ and column $l$ of the matrix. The length of this array is $n(n+1) / 2$, which is the number of distinct elements on or below the diagonal. Several routines described below have an array of this length in their list of arguments. In this storage format, the element in row $i$ and column $j$, where $i \geq j$, is in element $i(i-1) / 2+j-1$ of the array.

This array can be passed to the solver by using the subroutine

int SDPConeSetADenseVecMat(SDPCone sdpcone, int blockj, int vari, int $\mathrm{n}$, double alpha, double val[], int nnz);

The first argument point to a semidefinite cone object, the second argument specifies the block number $j$, and the third argument specifies the variable $i$ associated with it. Variables $1, \ldots, m$ correspond to matrices $A_{1, j}, \ldots, A_{m, j}$, whereas variable 0 corresponds to $C_{j}$. The fourth argument is the dimension (number of rows or columns) of the matrix, $n$. The sixth argument is the array, and the seventh argument is the length of the array. The data array will be multiplied by the scalar in the fifth argument. The application is responsible for allocating this array of data and eventually freeing it. SDPCone will directly access this 
array in the course of the solving the problem, so it should not be freed until the solver is finished.

A matrix can be passed to the solver in sparse format by using the subroutine

int SDPConeSetASparseVecMat(SDPCone sdpcone, int blockj, int vari, int $n$, double alpha, int ishift, const int ind [], const double val[], int nnz);

In this subroutine, the first five arguments are the same as in the subroutine for dense matrices. The seventh and eighth arguments are an array an integers and an array of double-precision variables. The final argument states the length of these two arrays, which should equal the number of nonzeros in the lower triangular part of the matrix. The array of integers specifies which elements of the array (10) are included in the array of doubles. For example, the matrix

$$
A_{i, j}=\left[\begin{array}{ccc}
3 & 2 & 0 \\
2 & 0 & 6 \\
0 & 6 & 0
\end{array}\right]
$$

could be inserted into the cone using one of several ways. If the first element in the val array is $a_{1,1}$, the first element in the ind array should be 0 . If the second element in the val array is $a_{3,2}$, then the second element in ind array should be 4 . When the ordering of elements begins with 0 , as just shown, the fifth argument ishift in the subroutine should be set to 0 . In general, the argument ishift specifies the index assigned to $a_{1,1}$. Although the relative ordering of the elements will not change, the indices assigned to them will range from ishift to ishift $+n(n+1) / 2-1$. Many applications, for instance, prefer to index the array from 1 to $n(n+1) / 2$, setting the index argument to 1 . The matrix (11) can be set in the block $j$ and variable $i$ of the semidefinite cone by using one of the routines

SDPConeSetASparseVecMat (sdpcone, $j, i, 3,1.0,0$, ind1, val1, 3) ;

SDPConeSetASparseVecMat (sdpcone, $j, i, 3,1.0,1$, ind2, val2 , 3);

SDPConeSetASparseVecMat (sdpcone, $j, i, 3,1.0,3$, ind3, val3 , 4) ;

SDPConeSetASparseVecMat (sdpcone, $j, i, 3,0.5,0$, ind4, val4, 3);

where

$$
\begin{array}{rlrl}
\text { ind1 } & =\left[\begin{array}{lll}
0 & 1 & 4
\end{array}\right] ; & & \operatorname{val1}=\left[\begin{array}{lll}
3 & 2 & 6
\end{array}\right] ; \\
\text { ind2 }=\left[\begin{array}{llll}
1 & 2 & 5
\end{array}\right] ; & \operatorname{val2}=\left[\begin{array}{lll}
3 & 2 & 6
\end{array}\right] ; \\
\text { ind3 }=\left[\begin{array}{llll}
7 & 3 & 5 & 4
\end{array}\right] ; & \operatorname{val3}=\left[\begin{array}{llll}
6 & 3 & 0 & 2
\end{array}\right] ; \\
\text { ind4 }=\left[\begin{array}{lll}
0 & 1 & 4
\end{array}\right] ; & \operatorname{val4}=\left[\begin{array}{lll}
6 & 4 & 12
\end{array}\right] ;
\end{array}
$$

As these examples suggest, there are many other ways to represent the sparse matrix. The nonzeros in the matrix do not have to be ordered, but ordering them may improve the efficiency of the solver. SDPCone assumes that all matrices $A_{i, j}$ and $C_{j}$ that are not explicitly defined and passed to the SDPCone structure will equal the zero matrix. Furthermore, there exist routines SDPConeAddASparseVecMat and SDPConeAddADenseVecMat that can be used to write a constraint matrix as a sum of multiple matrices. The arguments to these functions match those of the corresponding SDPConeSetASparseVecMat routines.

To check whether the matrix passed into the cone matches the one intended, one can use the subroutine 
int SDPConeViewDataMatrix(SDPCone sdpcone, int blockj, int vari);

to print the matrix to the screen. The output prints the row and column numbers, indexed from 0 to $n-1$, of each nonzero element in the matrix. The subroutine int SDPConeView (SDPCone sdpcone, int blockj) can be used to view all of the matrices in a block.

After the DSDP solver has been applied to the data and the solution matrix $X_{j}$ have been computed (see DSDPComputeX), the matrix can be accessed by using the command

int SDPConeGetXArray (SDPCone sdpcone, int blockj, double *xmat [], int *nn);

The third argument is the address of a pointer that will be set to the array containing the solution. The integer whose address is passed in the fourth argument will be set to the length of this array, $n(n+1) / 2$, for the packed symmetric storage format. Since the $X$ solutions are usually fully dense, no sparse representation is provided. These arrays were allocated by the SDPCone object during DSDPSetup, and the memory will be freed by the DSDP solver object when it is destroyed. The array used to store $X_{j}$ could be overwritten by other operations on the SDPCone object. The command

int SDPConeComputeX (SDPCone sdpcone, int blockj, int $\mathrm{n}$, double xmat [], int $\mathrm{nn}$ );

recomputes the matrix $X_{j}$ and places it into the array specified in the fourth argument. The length of this array is the fifth argument and the dimension of the block in the third argument. The vectors $y$ and $\Delta y$ needed to compute the matrices $X_{j}$ are stored internally in SDPCone object. The subroutine

int SDPConeViewX(SDPCone sdpcone, int blockj, int $n$, double xmat[], int $n n$ );

can be used to print this matrix to standard output.

The dimension of each block can be found by using the routine

int SDPConeGetBlockSize(SDPCone sdpcone, int blockj, int $* n$ );

where the second argument is the block number and the third argument is the address of an integer variable.

The inner product of $X_{j}$ with $C_{j}, A_{i, j}$, and $I_{j}$ can be computed by using the routine

int SDPConeAddADotX (SDPCone sdpcone, int blockj, double alpha, double xmat [], int $\mathrm{nn}$, double $\operatorname{adotx}[]$, int $\mathrm{mp} 2)$;

The second argument specifies which block to use, and the third argument is a scalar that will be multiplied by the inner products. The fourth argument is the array containing $X_{j}$, and the fifth argument is the length of the array. The sixth argument is an array of length $m+2$, and the seventh argument should equal $m+2$, where $m$ is the number of variables in $y$. This routine will add alpha times $\left\langle C_{j}, X_{j}\right\rangle$ to the initial element of the array, alpha times $\left\langle A_{i, j}, X_{j}\right\rangle$ to element $i$ of the array, and alpha times $\left\langle I_{j}, X_{j}\right\rangle$ to last element of the array.

The matrix $S$ in (D) can be computed and copied into an array by using the command 
int SDPConeComputeS(SDPCone sdpcone, int blockj, double c, double y[], int m, double $r$, int $\mathrm{n}$, double smat [], int $\mathrm{nn}$ );

The second argument specifies which block to use, and the fourth argument is an array containing the variables $y$. The third argument is the multiple of $C$ to be added, and the sixth argument is the multiple of the identity matrix to be added. The sixth argument is the dimension of the block, and the seventh argument is an array for $S$ whose length is given in the eighth argument.

Special support for combinatorial applications also exists. In particular, the subroutines

int SDPConeComputeXV(SDPCone sdpcone, int blockj, int *dpsdefinite);

int SDPConeXVMultiply(SDPCone sdpcone, int blockj, double v1[], double v2[], int $\mathrm{n}$ );

int SDPConeAddXVAV(SDPCone sdpcone, int blockj, double $\mathrm{v}[]$, int $\mathrm{n}$, double vav[], int $\mathrm{mp} 2$ );

support the use of randomized algorithms for combinatorial optimization. The first routine computes a matrix $V_{j}$ such that $X=V_{j} V_{j}^{T}$. The second routine computes the matrixvector product $w=V_{j} v$, where $w$ and $v$ are vectors of dimension equal to the dimension of the block. The third routine computes the vector-matrix-vector product $v^{T} A_{i, j} v$ for $C$, $A_{1, j}, \ldots, A_{m, j}$ and the identity matrix. The length of the array in the fifth argument is $m+2$ and should be set in the final argument. In these applications, use of the routine DSDPConeComputeX may not be necessary if the full matrix is not required.

The memory required for the $X_{j}$ matrix can be significant for large problems. If the application has an array of double-precision variables of length $n(n+1) / 2$ available for use by the solver, the subroutine

int SDPConeSetXArray(SDPCone sdpcone, int blockj, int $\mathrm{n}$, double xmat [], int $\mathrm{nn}$ );

can be used to pass it to the cone. The second argument specifies the block number whose solution will be placed into the array xmat. The third argument is the dimension of the block. The dimension specified in the fifth argument $\mathrm{nn}$ refers to the length of the array. The SDPCone object will use this array as a buffer for its computations and store the solution $X$ in this array at its termination. The application is responsible for freeing this array after the solution has been found.

DSDP also supports the symmetric full storage format. In symmetric full storage format, an $n \times n$ matrix is stored in an array of $n^{2}$ elements in row major order. That is, the elements of a matrix with $n$ rows and columns are ordered

$$
\left[\begin{array}{lllllllllllll}
a_{1,1} & 0 & \ldots & 0 & a_{2,1} & a_{2,2} & 0 & \ldots & 0 & \ldots & a_{n, 1} & \ldots & a_{n, n}
\end{array}\right] .
$$

The length of this array is $n \times n$. Early versions of DSDP 5 used the packed format exclusively, but this format has been added because it is more convenient for some applications. The routines

int SDPConeSetStorageFormat (SDPCone sdpcone,int blockj, char UPLQ);

int SDPConeGetStorageFormat (SDPCone sdpcone, int blockj, char *UPLQ); 
set and get the storage format for a block, respectively. The second argument specifies the block number and the third argument should be ' $P$ ' for packed storage format or ' $U$ ' for full storage format. The default value is ' $\mathrm{P}$ '. These storage formats correspond to the LAPACK storage formats for the upper half matrices in column major ordering. All of the above commands apply to the symmetric full storage format. One difference in their use, however, is that the size of the arrays in the arguments should be $n \times n$ instead of $n \times(n+1) / 2$. With the symmetric full storage format, if the first element in the val array is $a_{1,1}$, the first element in the ind array should be 0 . If the second element in the val array is $a_{3,2}$, then the second element in ind array should be 8 . The matrix (11) can be set in the block $j$ and variable $i$ of the semidefinite cone by using the routine

int SDPConeSetASparseVecMat (sdpcone, $j, i, 3,1.0,0$, ind1, val1,3);

where

$$
\text { ind1 }=\left[\begin{array}{lll}
0 & 3 & 7
\end{array}\right] ; \quad \operatorname{val1}=\left[\begin{array}{lll}
3 & 2 & 6
\end{array}\right] ; \text {; }
$$

\subsection{LP Cone}

To specify an application with a cone of linear scalar inequalities, one can use the subroutine int DSDPCreateLPCone( DSDP dsdp, LPCone *newlpcone);

to create a new object that describes a cone with 1 or more linear scalar inequalities. The first argument is an existing DSDP object, and the second argument is the address of an LPCone variable. This subroutine will allocate structures needed to specify the constraints and point the variable to this structure. Multiple cones for these inequalities can be created for the same DSDP object, but it is usually more efficient to group all inequalities of this type into the same structure. All subroutines that pass data to the LP cone require an LPCone object in the first argument.

A list of $n$ linear inequalities in (D) is passed to the object in sparse column format. The vector $c \in \mathbb{R}^{n}$ should be considered an additional column of the data. (In the formulation (P), the data $A$ and $c$ are represented in sparse row format.)

The data is passed to the LPCone using the subroutine

int LPConeSetData(LPCone lpcone, int $\mathrm{n}$, const int nnzin[], const int row [], const double aval[]);

In this case, the integer array nnzin has length $m+2$, and begins with 0 , and nnzin [i+1]nnzin [i] equals the number of nonzeros in column $i(i=0, \ldots, m)$ (or row $i$ of $A$ ). The length of the second and third array equals the number of nonzeros in $A$ and $c$. The arrays contain the nonzeros and the associated row numbers of each element (or column numbers in $A$ ). The first column contains the elements of $c$, the second column contains the elements corresponding to $y_{1}$, and the last column contains elements corresponding to $y_{m}$.

For example, consider the following problem in the form of (D):

$$
\begin{array}{llll}
\text { Maximize } & y_{1}+y_{2} \\
\text { Subject to } & 4 y_{1}+2 y_{2} \leq 6 \\
& 3 y_{1}+7 y_{2} \leq 10 \\
& & -y_{2} \leq 12
\end{array}
$$


This example has three inequalities, so the dimension of the $x$ vector would be 3 and $n=3$. The input arrays would be as follows:

$$
\begin{aligned}
& \text { nnzin }=\left[\begin{array}{llll}
0 & 3 & 5 & 7
\end{array}\right] \\
& \text { row }=\left[\begin{array}{llllllll}
0 & 1 & 2 & 0 & 1 & 0 & 1 & 2
\end{array}\right] \\
& \text { aval }=\left[\begin{array}{llllllll}
6.0 & 10.0 & 12.0 & 4.0 & 3.0 & 2.0 & 7.0 & -1.0
\end{array}\right]
\end{aligned}
$$

An example of the use of this subroutine can be seen in the DSDPROOT/examples/readsdpa.c.

If it is more convenient to specify the vector $c$ in the last column, consider using the subroutine:

int LPConeSetData2 (LPCone lpcone, int $\mathrm{n}$, const int ik[], const int cols[], const double vals[]);

This input is also sparse column input, but the $c$ column comes last. In this form the input arrays would be as follows:

$$
\begin{aligned}
& \text { nnzin }=\left[\begin{array}{llll}
0 & 2 & 5 & 7
\end{array}\right] \\
& \text { row }=\left[\begin{array}{llllllll}
0 & 1 & 0 & 1 & 2 & 0 & 1 & 2
\end{array}\right] \\
& \text { aval }=\left[\begin{array}{llllllll}
4.0 & 3.0 & 2.0 & 7.0 & -1.0 & 6.0 & 10.0 & 12.0
\end{array}\right]
\end{aligned}
$$

This subroutine is used in the DSDP Matlab mex function, which can be used as an example.

The subroutines

int LPConeView (LPCone lpcone);

int LPConeView2(LPCone lpcone);

can be used to view the data that has been set and verify the correctness of the data. Multiple LPCone structures can be used, but for efficiency, it is often better to include all linear inequalities in a single cone.

The variables $s$ in (D) and $x$ in $(\mathrm{P})$ can be found by using the subroutines

int LPConeGetXArray (LPCone lpcone, double *xout [], int $* n$ );

int LPConeGetSArray (LPCone lpcone, double $*$ sout [], int $* n$ );

In these subroutines, the second argument sets a pointer to an an array of doubles containing the variables, and the integer in the third argument is set to the length of this array. These array are allocated by the LPCone object, and the memory is freed when the DSDP object is destroyed. Alternatively, the application can give the LPCone an array in which to put the solution $x$ of $(\mathrm{P})$. This array should be passed to the cone by using the following subroutine:

int LPConeSetXVec(LPCone lpcone, double xout [], int $\mathrm{n}$ );

At completion of the DSDP solver, the solution $x$ will be copied into this array xout, which must have length $n$. The slack variables $s$ may be scaled. To get the unscaled vector, pass an array of appropriate length into the object using int LPConeGetSArray2 (LPCone Ipcone, double $\mathrm{s}[]$, int $\mathrm{n}$ ). This subroutine will copy the slack variables into the array.

A special type of semidefinite and LP cone contains only simple bounds on the variables $y$. Corresponding to lower and upper bounds on the $y$ variables are surplus and slack variables in $(\mathrm{P})$ with a cost. The subroutine 
int DSDPCreateBCone(DSDP dsdp, BCone *bcone);

will create this cone from an existing DSDP object and point the BCone variable to the new structure. The bounds on the variables can be set by using the subroutines

int BConeSetLowerBound(BCone bcone, int vari, double yi);

int BConeSetUpperBound(BCone bcone, int vari, double yi);

The first argument is the conic object, the second argument identifies a variable from 1 to $m$, and the third argument is the bound. Here $m$ is the total number of variables in the vector $y$. For applications using the formulation $(\mathrm{P})$, the subroutines

int BConeSetPSurplusVariable(BCone bcone, int vari);

int BConeSetPSlackVariable(BCone bcone, int vari);

may be a more convenient way to represent an inequality in $(\mathrm{P})$. These commands are equivalent to setting a lower or upper bound on a variable $y_{i}$ to zero. To improve the memory allocation process, the application may use the subroutines int BConeAllocateBounds ( BCone bcone, int nbounds); to tell the object how many bounds will be specified. This subroutine is optional, but it may improve the efficiency of the memory allocation. The subroutine int BConeSetXArray (BCone, double xout [], int $\mathrm{n}$ ) will set an array in the cone where the cone will copy the variables $x$. To view the bounds, the application may use the subroutine int BConeView (BCone bcone). To retrieve the dual variables $x$ corresponding to these constraints, one can use the subroutine int BConeCopyX (BCone bcone, double $\mathrm{xl}[]$, double $\mathrm{xu}[]$, int $\mathrm{m}$ ). The second and third arguments of this routine are an array of length $m$, the fourth argument. This routine will set the values of this array to the value of the corresponding variable. When no bound is present, the variable will equal zero.

In some applications it may be useful to fix a variable to a number. Instead of modeling this constraint as a pair of linear inequalities, fixed variables can be passed directly to the solver by using the subroutine

int DSDPSetFixedVariables(DSDP dsdp, double vars[], double vals[], double $\mathrm{x}[]$, int $\mathrm{n}$ );

In this subroutine, the array of variables in the second argument is set to the values in the array of the third argument. The fourth argument is an optional array in which the solver will put the sensitivities to these fixed variables. The final argument is the length of the arrays. Note that the values in the second argument are integer numbers from 1 to $m$ represented in double precision. Again, the integers should be one of $1, \ldots, m$. Alternatively, a single variable can be set to a value by using the subroutine int DSDPFixVariable (DSDP dsdp, int vari, double val).

\subsection{Applying the Solver}

After setting the data associated with the constraint cones, DSDP allocates internal data structures and factors the data in the subroutine

int DSDPSetup(DSDP dsdp); 
This subroutine factors the data, creates a Schur complement matrix with the appropriate sparsity, and allocates additional resources for the solver. This subroutine should be called after setting the data but before solving the problem. Furthermore, it should be called only once for each DSDP object. On very large problems, insufficient memory on the computer may be encountered in this subroutine, so the error code should be checked. The subroutine

int DSDPSetStandardMonitor(DSDP dsdp, int $\mathrm{k}$ );

will tell the solver to print the objective values and other information at each $\mathrm{k}$ iteration to standard output. The subroutine int DSDPLogInfoAllow(int,0) will print even more information if the first argument is positive.

The subroutine

int DSDPSolve(DSDP dsdp);

attempts to solve the problem. This subroutine can be called more than once. For instance, the user may try solving the problem by using different initial points.

After solving the problem, the subroutine

int DSDPComputeX (DSDP dsdp);

can be called to compute the variables $X$ in $(\mathrm{P})$. These computations are not performed within the solver because these variables are not needed to compute the step direction.

Each solver created should be destroyed with the command

int DSDPDestroy(DSDP dsdp);

This subroutine frees the work arrays and data structures allocated by the solver.

\subsection{Convergence Criteria}

Convergence of the DSDP solver may be defined by using several options. The precision of the solution can be set by using the subroutine

int DSDPSetGapTolerance(DSDP dsdp, double rgaptol);

The solver will terminate if there is a sufficiently feasible solution such that the difference between the objective values in (DD) and (PP), divided by the sum of their absolute values, is less than the prescribed number. A tolerance of 0.001 provides roughly three digits of accuracy, whereas a tolerance of $1.0 \mathrm{e}-5$ provides roughly five digits of accuracy. The subroutine

int DSDPSetMaxIts(DSDP dsdp, int maxits);

specifies the maximum number of iterations. The subroutine int DSDPSetDualBound( DSDP, double) specifies an upper bound on the objective value in (D). The algorithm will terminate when it finds a point when the variable $r$ in (DD) is less than the prescribed tolerance and the objective value in (DD) is greater than this number. 


\subsection{Detecting Infeasibility}

Infeasibility in either $(\mathrm{P})$ or $(\mathrm{D})$ can be determined by using the subroutine int DSDPGetSolutionType(DSDP dsdp, DSDPSolutionType *pdfeasible);

This command sets the second argument to an enumerated type. There are four types for DSDPSolutionType:

- The type DSDP_PDFEASIBLE means that both (D) and (P) have feasible solutions and their objective values are bounded.

- The type DSDP_UNBOUNDED means that $(\mathrm{D})$ is unbounded and $(\mathrm{P})$ is infeasible. This type applies when the variable $r \leq \epsilon_{r}$ and $\|\mathcal{A} X-b\|_{\infty} / \operatorname{trace}(X)>\epsilon_{P}$. In this case, at least one variable $y_{i}$ will be near its bound. The subroutine DSDPSetYBounds can adjust these bounds if the user thinks that they are not big enough to permit feasibility. Large bounds may create numerical difficulties in the solver, but they may also permit feasible solutions and improve the quality of the certificate of infeasibility. Normalizing the vector $y$ will provide an approximate certificate of infeasibility for $(\mathrm{P})$.

- The type DSDP_INFEASIBLE means that $(\mathrm{D})$ is infeasible and $(\mathrm{P})$ is unbounded. This type applies when the variable $r>\epsilon_{r}$ and $\|\mathcal{A} X-b\|_{\infty} / \operatorname{trace}(X) \leq \epsilon_{P}$. In this case, the trace of the variables $X$ in $(\mathrm{P})$ will be near the bound $\Gamma$. The subroutines DSDPSetPenaltyParameter can adjust $\Gamma$ if the user thinks that it is too small, A larger parameter may create numerical difficulties in the solver, but it may also improve the quality of the certificate of infeasibility. Normalizing these variables so that to have a trace of 1.0 will provide an approximate certificate of infeasibility.

- The type DSDP_PDUNKNOWN means DSDP was unable to determine feasibility in either solution. This type applies when the initial point for (DD) was infeasible or if the bounds on $y$ appear to be too small to permit a feasible solution.

The tolerance $\epsilon_{r}$ can be set by using the subroutine int DSDPSetRTolerance (DSDP, double). The tolerance $\epsilon_{P}$ can be set using the subroutine int DSDPSetPTolerance (DSDP, double). The subroutines int DSDPGetRTolerance(DSDP, double*) and int DSDPGetPTolerance( DSDP, double*) can be used to get the current tolerances.

\subsection{Solutions and Statistics}

The objective values in (PP) and (DD) can be retrieved by using the commands

int DSDPGetPPObjective(DSDP dsdp, double *pobj);

int DSDPGetDDObjective(DSDP dsdp, double $*$ dobj);

The second argument in these routines is the address of a double-precision variable.

The solution vector $y$ can be viewed by using the command

int DSDPGetY(DSDP dsdp, double y[], int $\mathrm{m}$ ); 
The user passes an array of size $m$ where $m$ is the number of variables in the problem. This subroutine will copy the solution into this array.

The success of DSDP can be interpreted with the command

int DSDPStopReason(DSDP dsdp, DSDPTerminationReason *reason);

This command sets the second argument to an enumerated type. The various reasons for termination are listed below.

DSDP_CONVERGED The solutions to (PP) and (DD) satisfy the convergence criteria.

DSDP_MAX_IT The solver applied the maximum number of iterations without finding a solution.

DSDP_INFEASIBLE_START The initial point in (DD) was infeasible.

DSDP_INDEFINITE_SCHUR Numerical issues created an indefinite Schur matrix that prevented further progress.

DSDP_SMALL_STEPS Small step sizes prevented further progress.

DSDP_NUMERICAL_ERROR Numerical issues prevented further progress.

The subroutines

int DSDPGetBarrierParameter(DSDP dsdp, double *mu);

int DSDPGetR(DSDP dsdp, double $* r$ );

int DSDPGetStepLengths (DSDP dsdp, double *pstep, double *dstep);

int DSDPGetPnorm(DSDP dsdp, double *pnorm);

provide more information about the current solution. The subroutines obtain the barrier parameter, the variable $r$ in (DD), the step lengths in (PP) and (DD), and a distance to the central path at the current iteration.

A history of information about the convergence of the solver can be obtained with the commands

int DSDPGetGapHistory(DSDP dsdp, double gaphistory[], int history);

int DSDPGetRHistory(DSDP dsdp, double rhistory[], int history);

These subroutines retrieve the history of the duality gap and the variable $r$ in (DD) for up to 100 iterations. The user passes an array of double-precision variables and the length of this array. The subroutine

int DSDPGetTraceX(DSDP dsdp, double *tracex);

gets the trace of the solution $X$ in $(\mathrm{P})$. Recall that the penalty parameter must exceed this quantity in order to return a feasible solution from an infeasible starting point. The subroutine

int DSDPEventLogSummary(void)

will print a summary of time spent in each cone and many of the primary computational subroutines. 


\subsection{Improving Performance}

The performance of the DSDP may be significantly improved with the proper selection of bounds, parameters, and initial point.

The application may specify an initial vector $y$ to (D), a multiple of the identity matrix to make the initial matrix $S$ positive definite, and an initial barrier parameter. The subroutine int DSDPSetYO(DSDP dsdp, int vari, double yi0) can specify the initial value of the variable $y_{i}$. Like the objective function in $(\mathrm{D})$, the variables are labeled from 1 to $\mathrm{m}$. By default the initial values of $y$ equal 0. Since convergence of the algorithm depends on the proximity of the point to the central path, initial points can be difficult to determine. Nonetheless, the subroutine

int DSDPSetRO(DSDP dsdp, double ro);

will set the initial value of $r$ in (DD). If $r 0<0$, a default value of $r 0$ will be chosen. If $S^{0}$ is not positive definite, the solver will terminate with an appropriate termination flag. The default value is usually very large (1e10), but smaller values can significantly improve performance.

The subroutine int DSDPSetPotentialParameter(DSDP dsdp, double rho) sets the potential parameter $\rho$. This parameter must be greater than 1 . The default value is 4.0, but larger values such as 5 or 10 can significantly improve performance. Feasibility in $(\mathrm{D})$ is enforced by means of a penalty parameter. By default it is set to $10 e 8$, but other values can affect the convergence of the algorithm. This parameter can be set by using int DSDPSetPenaltyParameter(DSDP dsdp, double Gamma), where Gamma is the large positive penalty parameter $\Gamma$. This parameter must exceed the trace of the solution $X$ in order to return a feasible solution from an infeasible starting point. The subroutine int DSDPUsePenalty (DSDP dsdp, int yesorno) is used to modify the algorithm. By default, the value is 0 . A positive value means that the variable $r$ in (DD) should be kept positive, treated like other inequalities, and penalized with the parameter Gamma. The subroutine int DSDPSetZBar(DSDP dsdp, double zbar) sets an initial upper bound on the objective value at the solution. This value corresponds to the objective value of any feasible point of (PP). The subroutine int DSDPSetBarrierParameter(DSDP dsdp, double mu0) sets the initial barrier parameter. The default heuristic is very robust, but performance can get generally be improved by providing a smaller value.

DSDP applies the same Schur complement matrix for multiple linear systems. This feature often reduces the number of iterations and improves robustness. The cost of each iteration increases, especially when the dimension of the semidefinite blocks is of similar dimension to or larger than the number of variables $y$. The subroutine

int SDPConeSetParameter(SDPCone sdpcone, DSDP dsdp);

sets an appropriate parameter based upon the dimension of the semidefinite blocks. To manually set this parameter, the subroutine int DSDPReuseMatrix (DSDP dsdp, int reuse) can set a maximum on the number of times the Schur complement matrix is reused. The default value is 4, although the Matlab mex function and SDPA file reader set this parameter between 0 and 15 depending on the size of the semidefinite blocks and the number of variables $y$. Applications whose semidefinite blocks are small relative to the number of variables $y$ should probably use larger values, while applications whose semidefinite blocks have 
size equal to or greater than the number of variables should probably set this parameter to zero.

The convergence of the dual-scaling algorithm assumes the existence of a strict interior in both $(\mathrm{P})$ and $(\mathrm{D})$. The use of a penalty parameter can add an interior to (D). An interior to $(\mathrm{P})$ can be created by bounding the variables $y$. Default bounds of $-1 \mathrm{e} 7$ and $1 \mathrm{e} 7$ have been set, but applications may change these bounds by using the subroutine

int DSDPSetYBounds(DSDP dsdp, double minbound, double maxbound);

The second argument should be a negative number that is a lower bound of each variable $y_{i}$, and the third argument is an upper bound of each variable. These bounds should not be tight. If one of the variables nearly equals the bound at the solution, the solver will return a termination code saying (D) is unbounded. To remove these bounds, set both the lower and upper bound to zero.

\subsection{Iteration Monitor}

A standard monitor that prints out the objective value and other relevant information at the current iterate can be set by using the command

int DSDPSetStandardMonitor(DSDP dsdp, int k);

A user can write a customized subroutine of the form

int (*monitor) (DSDP dsdp, void* ctx);

This subroutine will be called from the DSDP solver each iteration. It is useful for writing a specialized convergence criteria or monitoring the progress of the solver. The objective value and other information can be retrieved from the solver by using the commands in the section 8.7. To set this subroutine, use the command

int DSDPSetMonitor(DSDP dsdp, int (*monitor)(DSDP, void*), void* ctx);

In this subroutine, the first argument is the solver, the second argument is the monitoring subroutine, and the third argument will be passed as the second argument in the monitoring subroutine. Examples of two monitors can be found in DSDPROOT/src/solver/dsdpconverge.c. The first monitor prints the solver statistics at each iteration, and the second monitor determines the convergence of the solver. A monitor can also be used to print the time, duality gap, and potential function at each iteration. Monitors have also been used to stop the solver after a specified time limit and change the parameters in the solver. 


\section{PDSDP}

The DSDP package can also be run in parallel on multiple processors. In the parallel version, the Schur complement matrix is computed and solved in parallel. The parallel Cholesky factorization in PDSDP is performed by using SCALAPACK. The parallel Cholesky factorization in SCALAPACK uses a two-dimensional block cyclic structure to distribute the data. The blocking parameter in SCALAPACK determines how many rows and columns are in each block. Larger block sizes can be faster and reduce the overhead of passing messages, but smaller block sizes balance the work among the processors more equitably. PDSDP used a blocking parameter of 32 after experimenting with several choices. Since SCALAPACK uses a dense matrix structure, this version is not appropriate when the Schur complement matrix is sparse.

The following steps should be used to run an application in parallel using PDSDP.

1. Install DSDP. Edit DSDPROOT/make. include to set the appropriate compiler flags.

2. Install SCALAPACK. This package contains parallel linear solvers and is freely available to the public.

3. Go to the directory DSDPROOT/src/pdsdp/scalapack/ and edit Makefile to identify the location of the DSDP and SCALAPACK libraries.

4. Compile the PDSDP file pdsdpscalapack.c, which implements the additional operations. Then compile the executable readsdpa.c, which will read an SDPA file.

A PDSDP executable can be used much like the serial version of DSDP that reads SDPA files. Given a SDPA file such as truss1. dat-s, the command

mpirun -np 2 dsdp5 truss1.dat-s -log_summary

will solve the problem using two processors. Additional processors may also be used. This implementation is best suited for very large problems.

Use of PDSDP as a subroutine library is also similar to the use of the serial version of the solver. The application must create the solver and conic object on each processor and provide each processor with a copy of the data matrices, objective vector, and options. At the end of the algorithm, each solver has a copy of the solution. The routines to set the data and retrieve the solution are the same.

The few differences between the serial and parallel version are listed below.

1. All PDSDP programs must include the header file:

\#include pdsdp5scalapack.h

2. Parallel applications should link to the DSDP library, the SCALAPACK library, and the compiled source code in dsdpscalapack.c. Linking to the BLAS and LAPACK libraries is usually included while linking to SCALAPACK.

3. The application should initialize and finalize MPI. 
4. After creating the DSDP solver object, the application should call

int PDSDPUseSCALAPACKLinearSolver(DSDP dsdp);

5. The monitor should be set on only one processor, for example:

MPI_Comm_rank (MPI_COMM_WORLD, \&rank);

if $(r a n k==0)$ info = DSDPSetStandardMonitor (dsdp);

An example of the usage is provided in DSDPROOT/pdsdp/ScaLAPACK/readsdpa.c. Scalability of medium- and large-scale problems has been achieved on up to 64 processors. The corrector direction does not scale well, so consider reducing the number of these steps for large groups of processors. See [1] for more details.

Source code that uses the parallel conjugate gradient method in PETSc to solve the linear systems is also included in the distribution. 


\section{Data Structures and Parameters}

The DSDP solver computes $\Delta^{\prime} y, \Delta^{\prime \prime} y, \Delta_{\nu} y,\|P(\nu)\|,\langle X(\nu), S\rangle$, and other quantities using operations on vectors, the Schur matrix, and the cones. Vector operations include sums and inner products. Operations on the Schur matrix include inserting elements and factoring the matrix. Objects representing a cone implement routines for computing its dual matrix $S$ from $y$, evaluating the logarithmic barrier function, computing $\mathcal{A} S^{-1}$, and computing $M$. The solver object computes $M$, for example, by calling the corresponding operations on each cone and summing the results. The solver computes $\Delta^{\prime} y$ through calls to the Schur matrix object that can factor the matrix and solve systems of linear equations. Table 2 shows eight of the primary data structures used in DSDP, the operations they implement, and the other objects required to implement those operations. For dense matrix structures, DSDP uses BLAS and LAPACK to operate on the data.

The LP cone and SDP cone objects implement the same interface for cones, but the implementation of these operations depends on whether the cone is a semidefinite cone, linear programming cone, or another type. The solver structure operates on the cone objects without knowing whether it is an SDP cone or another type. DSDP uses opaque pointers and function pointers to achieve polymorphic behavior.

Table 3 summarizes the significant parameters, options, and default values. The most important of these options is the initial variable $r$. By default, DSDP selects a value much larger than required to make $S \in \hat{K}$. Computational experience indicates that large values are more robust than smaller values. DSDP then sets the initial values of $\bar{z}=1 e 10$ and $\nu=\left(\bar{z}-b^{T} y+\Gamma r\right) /\left(n \rho_{n}\right)$. Users can manually set $\bar{z}$ and $\nu$, but choices better than the defaults usually require insight into the solution. The number of corrector steps can also significantly improve performance. In some examples, corrector steps can reduce the number of iterations by half - although the impact in total computation time is not as significant. Computational experience suggests that the number of corrector steps should be between 0 and 12 , and the time spent in these steps should not exceed $30 \%$. 
Table 2: Summary of primary data structures and their functionality.

Solver: Implements an algorithm for linear and semidefinite programming. Operations: $\Delta^{\prime} y, \Delta^{\prime \prime} y, \Delta_{\nu} y, \Delta^{c} y,\|P(\nu)\|,\langle X(\nu), S\rangle$, reduce $\nu$.

Implementations: Dual-Scaling Algorithm.

Instances: one.

Requires: Vector, Cone, Schur.

Vector: Represents $y, \mathcal{A} S^{-1}, b, \Delta^{\prime} y, \Delta^{\prime \prime} y, \Delta_{\nu} y$, and other internal work vectors. Operations: sum, inner product, norm.

Implementations: dense one-dimensional array.

Instances: about two dozen.

Schur: Represents $M$, the Schur complement of Newton equations.

Operations: add to row, add to diagonal, factor, solve, vector-multiply.

Implementations: sparse, dense, parallel dense.

Instances: one.

Cone: Represents data $C$ and $A_{i}$.

Operations: check if $S \leftarrow C-\mathcal{A}^{*} y \in K$, ln $\operatorname{det} S, \mathcal{A} S^{-1}, M, X(\nu)$.

Implementations: SDP Cone, LP Cone, Bounds on $\mathrm{y}$, Variable $r \geq 0$.

Instances: three or more.

Requires: Vector, Schur.

SDP Cone requires: V Matrix, Data Matrices, S Matrix, DS Matrix.

SDP V Matrix: Represents $X, S^{-1} A_{i} S^{-1}$, and a buffer for $C-\mathcal{A}^{*} y$.

Operations: $V \leftarrow 0, V \leftarrow V+\gamma w w^{T}$, get array.

Implementations: dense.

Instances: one per block.

SDP Data Matrix: Represents a symmetric data matrix.

Operations: $V \leftarrow V+\gamma A,\langle V, A\rangle, w^{T} A w$, get rank, get eigenvalue/vector.

Implementations: sparse, dense, identity, low-rank.

Instances: up to $m+1$ per block.

SDP S Matrix: Represents $S$ and checks whether $X(\nu) \succ 0$.

Operations: $S \leftarrow V$, Cholesky factor, forward solve, backward solve, determinant. Implementations: sparse, dense.

Instances: two per block.

SDP DS Matrix: Represents $\Delta S$.

Operations: $\Delta S \leftarrow V, w \leftarrow \Delta S v$.

Implementations: dense, sparse, diagonal.

Instances: one per block. 
Table 3: Summary of important parameters and initial values.

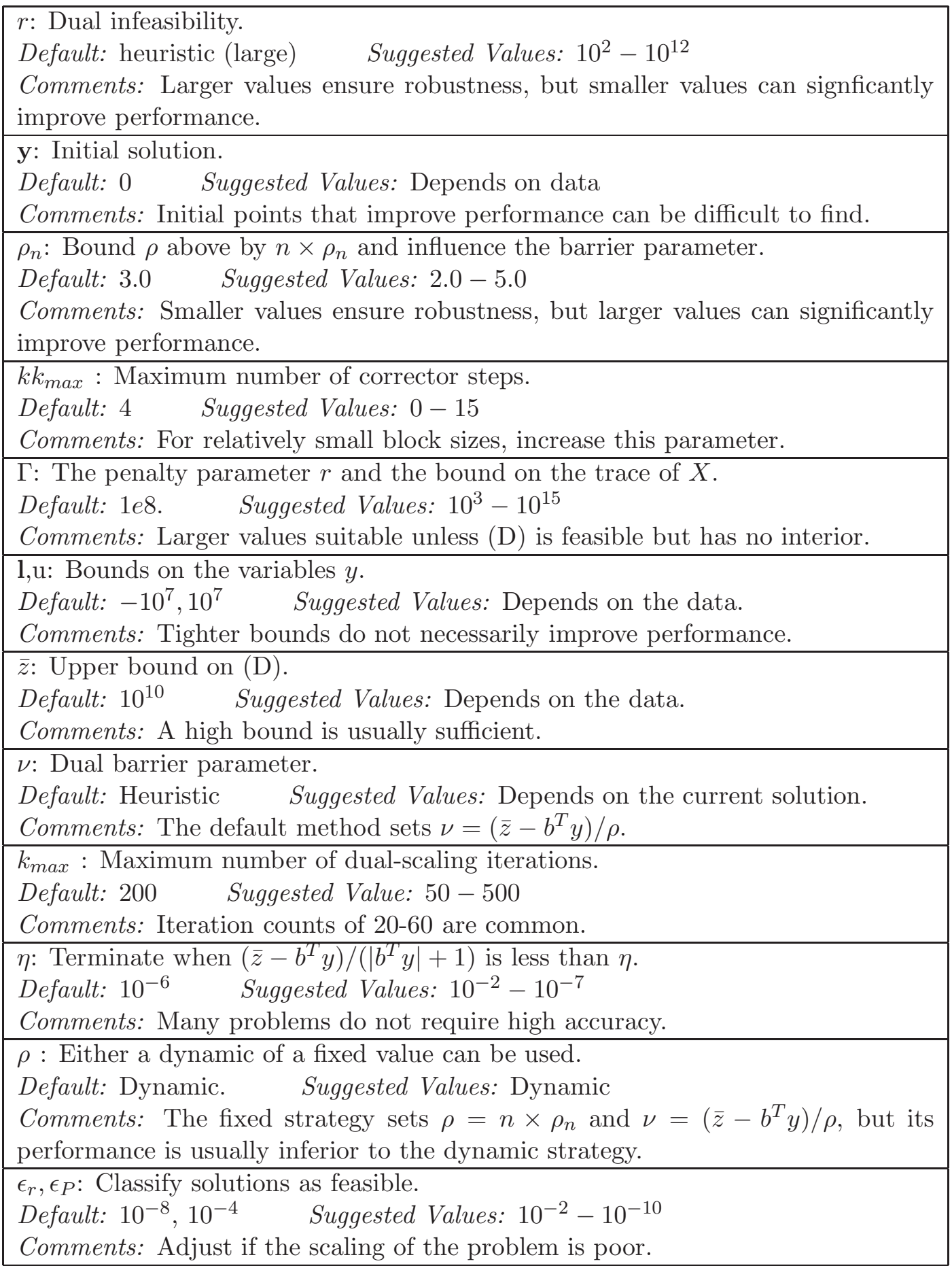




\section{Previous Versions}

DSDP began as a specialized solver for combinatorial optimization problems. Over the years, improvements in efficiency and design have enabled its use in many applications.

1997 At the University of Iowa the initial version of DSDPwas released. It solved the semidefinite relaxations of the maximum cut problem [3].

1999 DSDP version 2 increased functionality to address semidefinite cones with rank-one constraint matrices and LP constraints. It was used specifically for combinatorial problems such as minimum bisection, graph coloring, stable sets, and bound-constrained quadratic problems.

2000 DSDP version 3 was a preliminary implementation of a general purpose SDP solver that addressed applications from the Seventh DIMACS Implementation Challenge on Semidefinite and Related Optimization Problems. It ran in serial and parallel.

2002 DSDP version 4 added new sparse data structures to improve efficiency and precision. A Lanczos based line search and efficient iterative solver were added. It solved all problems in the SDPLIB collection that includes examples from control theory, truss topology design, and relaxations of combinatorial problems.

2004 DSDP version 5 [2] featured new data structures for semidefinite constraints, a corrector direction, and extensibility to structured applications in conic programming. Existence of the central path was ensured by bounding the variables.

\section{Acknowledgments}

We thank Xiong Zhang and Cris Choi for their help in developing this code. Xiong Zhang, in particular, was fundamental to the initial version of DSDP. We also thank Hans Mittelmann [4] for his efforts in testing and benchmarking the different versions of the code. We thank all of the users who have commented on previous releases and suggested improvements to the software. Their contributions have made DSDP a more reliable, robust, and efficient package. 


\section{References}

[1] Steven J. Benson. Parallel computing on semidefinite programs. Technical Report ANL/MCS-P939-0302, Mathematics and Computer Science Division, Argonne National Laboratory, March 2003.

[2] Steven J. Benson and Yinyu Ye. DSDP5: Software for semidefinite programming. Preprint ANL/MCS-P1289-0905, Mathematics and Computer Science Division, Argonne National Laboratory, September 2005.

[3] Steven J. Benson, Yinyu Ye, and Xiong Zhang. Solving large-scale sparse semidefinite programs for combinatorial optimization. SIAM Journal on Optimization, 10(2):443461, 2000 .

[4] Hans D. Mittelmann. Benchmarks for optimization software, 2005. $\mathrm{ftp}: / /$ plato.asu.edu/ftp/\{sdplib.txt, sparse_sdp.txt, dimacs.txt\}. 